\title{
ANÁLISIS ESPACIO-TEMPORAL DE LAS NOCHES CÁLIDAS EN EL LITORAL MEDITERRÁNEO DE ESPAÑA
}

\author{
Dominic ROYÉ 1 , Alberto MARTÍ EZPELETA² \\ ${ }^{1}$ Dep. de Geografia. Universidad de Cantabria. \\ ${ }^{2}$ Dep. de Geografia. Universidad de Santiago de Compostela. \\ dominic.roye@unican.es, alberto.marti@usc.es
}

\section{RESUMEN}

En este trabajo se aplica un nuevo enfoque al estudio de las noches calurosas, también denominadas "tropicales", en el litoral mediterráneo de España de cara a identificar aquellas noches en las que la población pueda verse afectada por estrés térmico. La utilización de un indicador obtenido a través de datos semihorarios ha permitido definir con más detalle las características térmicas de las noches veraniegas, pudiendo así evaluar con más precisión el riesgo para el bienestar y la salud de la población. Se produce un importante aumento de la frecuencia de noches tropicales y noches cálidas en el litoral mediterráneo, desde Cataluña hasta el sur de Andalucía. La menor latitud y la proximidad a la costa están relacionadas con la mayor persistencia del calor y del estrés térmico durante estas noches. En áreas de interior la persistencia es menor.

Palabras clave: noches tropicales, noches cálidas, Mediterráneo, España, salud humana.

\begin{abstract}
This paper presents a new methodology for the study of hot nights, also called "tropical nights", across the Mediterranean coast of Spain in order to identify those nights where people can be affected by heat stress. The use of one indicator obtained through half-hourly data has allowed to define in more detail the thermal characteristics of the nights, thereby being able to assess more accurately the risk to the health and well-being of the population. There is a significant increase in the frequency of tropical nights and hot nights on the Mediterranean coast, from Catalonia to the south of Andalusia. The lower latitude and proximity to the coastline are associated with greater persistence of heat and thermal stress during these nights. The hottest nights are more frequent and intense in the city center due to the effect of the urban heat island.
\end{abstract}

Key words: tropical nights, warm evenings, Mediterranean, Spain, human health

\section{INTRODUCCIÓN}

Los episodios de calor constituyen un fenómeno recurrente en los climas templados durante el periodo estival, especialmente en las regiones más meridionales y más expuestas a las masas de aire cálidas procedentes de latitudes subtropicales, causantes de dichos episodios. Se trata de un fenómeno que repercute negativamente tanto en 
el bienestar como en la salud de la población. Los efectos de las olas de calor en la población han sido descritos por diferentes autores, que han encontrado relaciones evidentes entre las altas temperaturas y la morbi-mortalidad (Bobb et al., 2014; Gronlund et al., 2014; Ye et al., 2012; Hajat \& Kosatky 2010). En la última década se ha podido observar a escala global un considerable aumento de estos episodios (Coumou y Robinson, 2013; Coumou y Rahmstorf, 2012). En el último informe del Grupo de Trabajo II del IPCC (2014) señala que hay evidencia de mayor mortalidad relacionada con el calor y menor con el frío en algunas zonas del planeta como Europa, y se advierte del muy probable incremento de la frecuencia e intensidad de los episodios de calor extremo que conllevará el calentamiento global. El IPCC destaca también como muy probable que los días y noches cálidos sean más numerosos y más cálidos, y no siempre asociados a las olas de calor.

La consecuencia más habitual de las noches calurosas sobre la salud es su impacto en el sueño y en el descanso de las personas. El calor puede llevar, entre otros efectos, a alteraciones y privaciones de éste debidos a la puesta en marcha de los procesos de termorregulación (Buguet, 2007). Concretamente, las temperaturas superiores a las del confort pueden influir en el aumento del desvelo y en la disminución de las fases REM (Rapid Eye Movement) y SWS (Slow-wave Sleep) (Haskella et al., 1981; Okamoto y Mizuno 2005, 2012). Las alteraciones del sueño se dan con mayor incidencia, al igual que el riesgo general de morbi-mortalidad durante los episodios de calor, en personas con avanzada edad (Buguet, 2007; Koppe et al., 2004).

Hay que recordar que en las ciudades los impactos negativos del calor sobre el confort y la salud se ven agravados por el fenómeno urbano de "isla de calor". Temperaturas más elevadas que en el entorno, favorecidas por diversos factores urbanos, y el más lento enfriamiento nocturno, generan durante los episodios de calor unas condiciones de mayor estrés térmico y el aumento del riesgo para la salud de los habitantes de las ciudades, en contraste con el medio rural circundante (Andrade y Alcoforado, 2008; Laaidi et al., 2012; Royé y Martí, 2015).

Teniendo en cuenta las proyecciones de cambio climático, en las que se estima una mayor frecuencia, duración e intensidad de los episodios de calor que afectarán a Europa, con mayores impactos en la península ibérica y en las regiones del Mediterráneo (Fischer \& Schär, 2010; IPCC, 2014), consideramos de interés poder disponer de indicadores adecuados que permitan evaluar el riesgo de impacto del calor nocturno sobre la salud y el bienestar de la población. Es por ello que el objetivo de este trabajo consiste en analizar con una metodología nueva el comportamiento espacio-temporal de las noches calurosas en el litoral mediterráneo.

\section{EL CONCEPTO DE "NOCHE TROPICAL"}

El concepto de "noche tropical" (NT) ha sido definido como una noche en la que la temperatura mínima es superior o igual a $\operatorname{los} 20^{\circ} \mathrm{C}$ (Vincent et al., 2005; Alexander et al., 2006; WMO, 2009; EEA Report, 2012; Donat et al., 2013; DWD, 2013). El Expert Team on Climate Change Detection and Indices utiliza los días con temperaturas mínimas $>20^{\circ} \mathrm{C}$ como uno de los indicadores climáticos para el seguimiento del cambio climático (Lisa et al., 2009; Russo y Sterl, 2011). Se trata de un índice de umbral, que pertenece a un grupo de índices en el que también se 
integran otros como días de fuerte precipitación o días de helada. En estos índices se fijan unos umbrales para identificar los días en los que se superan o no se alcanzan éstos. Como consecuencia, estos umbrales fijos no pueden ser válidos para todas las zonas climáticas del planeta (Alexander et al., 2006). En el caso de las noches tropicales es evidente que su umbral de $20^{\circ} \mathrm{C}$ puede ser útil en Europa o EEUU, pero para un clima tropical con temperaturas mínimas superiores a éstos durante varios meses, sería cuestionable. Un ejemplo ilustrativo al respecto de este problema puede verse en la página web del servicio meteorológico de Hong Kong (Hong Kong Observatory, 2012). En ella no se habla de "noches tropicales" sino de "hot nights"; y, en segundo lugar, el umbral aplicado se define como igual o superior a $28^{\circ} \mathrm{C}$. Incluso en un estudio sobre los efectos biometeorológicos de las temperaturas nocturnas de Atenas (Nastos y Matzarakis, 2008), se usó una temperatura mínima de $23^{\circ} \mathrm{C}$ como umbral. La mención de "tropical" es, pues, una referencia a una situación no habitual para las regiones extratropicales, como es el caso de Europa o gran parte de EEUU.

Se ha observado que el uso exclusivo de la temperatura mínima como referencia para delimitar las noches tropicales, puede resultar insuficiente para llevar a cabo un análisis detallado del impacto del calor nocturno en la salud (Royé \& Martí, 2015; Royé, 2015). Por una parte hay que tener en cuenta que la temperatura mínima suele alcanzarse habitualmente en los momentos próximos a la salida del sol; pero se pueden registrar temperaturas superiores a $20^{\circ} \mathrm{C}$ durante muchas horas de la noche y obtener, como registro mínimo, una temperatura inferior a $\operatorname{los} 20^{\circ} \mathrm{C}$. En estos casos el estrés térmico no es necesariamente menor que en los días con una temperatura mínima igual o superior a $20^{\circ} \mathrm{C}$, sobre todo si tenemos en cuenta que la fase inicial del sueño, en comparación con las siguientes, se describe como la más sensible y la que acumula las mayores alteraciones por estrés térmico (Okamoto et al., 2005). Estos autores demuestran la importancia de la temperatura en las primeras horas de la noche para el descanso durante las fases posteriores.

Pero también existe otro problema. Se han detectado diversos días en los que la temperatura mínima se ha producido antes de la media noche, en la franja comprendida entre las 23 y las $24 \mathrm{~h}$, aumentando la temperatura en las horas posteriores. En todos estos casos la temperatura mínima del día no sería un indicador adecuado de noche tropical ni de estrés térmico nocturno.

Estas limitaciones en el uso de la temperatura mínima como indicador de noches con riesgo de estrés térmico ha llevado a los autores Royé \& Martí (2015) a desarrollar y proponer un nuevo indicador de cara a mejorar la evaluación de este tipo de riesgo térmico.

\section{MÉTODOS}

Para utilizar el indicador propuesto es necesario disponer de datos horarios o subhorarios de la temperatura del aire (Ta). El índice de las Noches Cálidas (NC) o "Hot Nights" se calcula sumando el número de horas durante la noche en las que se supera el umbral de $20^{\circ} \mathrm{C}$. Posteriormente se relativiza el valor obtenido en función del número total de horas nocturnas para poder comparar las distintas noches del año, obteniendo así un porcentaje (Ecuación 1): 


$$
N C=\frac{\sum_{i} h_{i}}{\sum_{i} n_{i}} \times 100,
$$

donde: $h_{i}$ es el número de horas nocturnas del día i en las que $T a>=20^{\circ} \mathrm{C}$, y ni es el número total de horas nocturnas del día i.

El umbral térmico de los $20^{\circ} \mathrm{C}$ se estableció para poder hacer una comparación con el índice clásico de las Noches Tropicales. No obstante, para el cálculo de las noches cálidas, este umbral fijo ha sido sustituido por el percentil $95 \%$ de la temperatura mínima de cada lugar, con el objeto de adaptar el índice al clima local. La noche se define como el periodo comprendido entre la puesta y la salida del sol. Todo el proceso necesario para el cálculo del indicador se ha realizado con el entorno estadístico R-GNU (3.3). Para el cálculo de la variación del número de horas entre la puesta y la salida del sol, se ha usado el paquete Sun-methods \{maptools\}, que a su vez usa el algoritmo de la National Oceanic \& Atmospheric Administration (NOAA).

En una primera aproximación se ha establecido un mínimo del $40 \%$ de horas en las que se ha superado el percentil 95\% para clasificarlas como "noches cálidas", con el objetivo de diferenciarlas de las noches tropicales en las que el umbral se supera en el $100 \%$ de las horas nocturnas. La fijación del umbral en el $40 \%$ de las horas nocturnas se ha hecho teniendo en cuenta que las primeras fases del sueño son las más sensibles y las que afectan en mayor medida el descanso del organismo. Por ello, hemos considerado que aquellas noches en las que durante las primeras horas se supere este umbral, pueden generar estrés térmico y problemas en el sueño e, incluso, en la salud.

El área de estudio comprende el litoral mediterráneo español (Fig. 1), donde se aplica el indicador propuesto para analizar las posibles relaciones existentes entre la ocurrencia de noches cálidas y los factores geográficos, teniendo siempre al mar Mediterráneo como factor geográfico común. Las estaciones elegidas corresponden a los aeropuertos de Barcelona, Girona, Valencia, Palma de Mallorca, Alicante, Málaga y Almería. Los datos usados corresponden al Integrated Surface Global Hourly Data, accessibles desde la NOAA. Los registros de temperatura tienen un intervalo de 30 minutos. Los valores ausentes fueron estimados con una interpolación de splines a través de la función na.spline de la librería $\{z o o\}$.

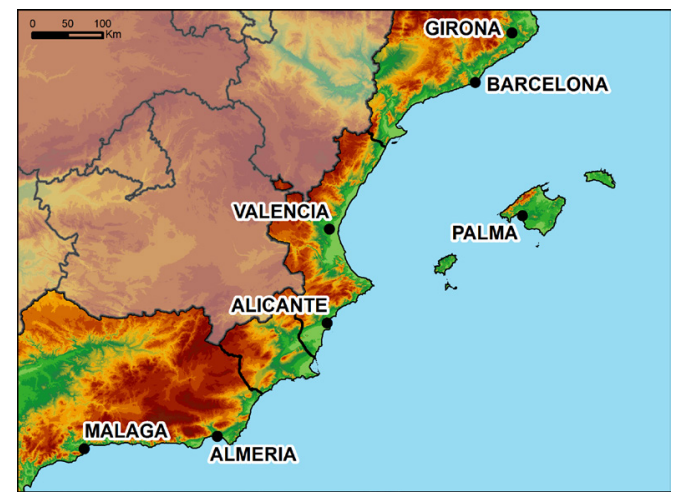

Fig. 1: Red de estaciones analizadas 


\section{RESULTADOS Y DISCUSIÓN}

El primer aspecto destacable a vista de los resultados (Tabla 1) es el elevado número de NT en todas las ciudades bañadas por el Mediterráneo, con valores que van desde las 28 noches anuales en Palma, pasando por las 43 de Barcelona, hasta las 81 noches que padecen en Almería, donde casi una de cada cuatro noches del año tienen carácter tropical. Girona, por su carácter interior, registra un escaso número medio anual de NT, tan solo 6. Como se puede observar en la tabla, estos valores están directamente relacionados con las temperaturas medias de las mínimas. Entre las ciudades que están situadas en la costa occidental peninsular se observa claramente un aumento del número de NT de norte a sur, desde Barcelona con 43 noches, pasando por Valencia con 54 y Alicante con 61 hasta Almería con 81 noches. Málaga, situada en la costa sur peninsular, con una latitud similar a la de Almería, registra sin embargo un número bastante inferior de NT a esta última. La explicación de este comportamiento espacial podría encontrarse en la combinación del factor latitudinal y la mayor exposición a las masas de aire cálido y seco procedentes del norte de África, sobre todo en verano, además de la influencia de la temperatura superficial del mar Mediterráneo.

\begin{tabular}{|c|c|c|c|c|c|c|}
\hline & $\begin{array}{l}\text { NT } \\
{[\mathrm{cv}]}\end{array}$ & $\begin{array}{c}\text { Rachas }^{1} \text { NT } \\
{[\mathrm{cv}]}\end{array}$ & $\begin{array}{l}\text { Percentil 95\% } \\
\text { (Tmin) }\end{array}$ & $\begin{array}{l}\mathrm{NC} \\
{[\mathrm{cv}]}\end{array}$ & $\begin{array}{c}\text { Rachas NC } \\
{[\mathrm{cv}]}\end{array}$ & $\begin{array}{c}\mathrm{T}^{\mathrm{a}} \text { media de las } \\
\text { mínimas }\left({ }^{\circ} \mathrm{C}\right)\end{array}$ \\
\hline Girona & $6[95]$ & $1,6[70]$ & 18,8 & $60[42]$ & $4,6[200]$ & 8,65 \\
\hline Barcelona & 43 [61] & $4,5[178]$ & 22 & 88 [14] & $7,0[176]$ & 11,91 \\
\hline Valencia & 54 [29] & 4,6 [167] & 22 & $83[21]$ & 4,5 [195] & 12,31 \\
\hline Palma & $28[60]$ & $2,4[110]$ & 20,7 & $62[24]$ & $4,6[161]$ & 10,8 \\
\hline Alicante & $62[23]$ & 7.5 [164] & 22,3 & 80 [17] & 6,2 [198] & 13,17 \\
\hline Almería & $81[19]$ & 7,5 [176] & 23,5 & 84 [16] & 5,3 [155] & 14,68 \\
\hline Málaga & $56[35]$ & $3,7[164]$ & 22 & 82 [21] & $5,1[191]$ & 13,48 \\
\hline
\end{tabular}

Tab. 1: Valores medios anuales de noches tropicales y noches cálidas (1974-2015). ${ }^{I}$ promedio de días consecutivos; $\mathrm{cv}=$ coeficiente de variación \%.

Durante los meses de verano, sobre todo entre julio y septiembre, las temperaturas de las aguas superficiales del Mediterráneo que bañan la costa oriental peninsular experimentan un gradiente de aumento entre el golfo de León y la costa de Alicante y Almería (Fig. 2), para volver a descender por el litoral del sur de la península hacia el estrecho de Gibraltar, debido a la mezcla de las aguas del Atlántico con las del Mediterráneo. Ello explica en buena parte que el número de NT en Málaga sea inferior al de Almería, a pesar de estar situadas a una misma latitud, pues las temperaturas del mar algo más frescas favorecen una mayor disminución de las temperaturas nocturnas del aire en las localidades costeras. Estas variaciones espaciales de norte a sur también se observan en las secuencias de noches tropicales consecutivas, siendo más persistentes en la mitad meridional de la costa oriental, donde las rachas alcanzan valores medios de 7,5 noches continuadas con mínimas superiores a los $20^{\circ} \mathrm{C}$, mientras que en Barcelona las secuencias medias son de 4,5 noches. Esta estrecha interacción entre el mar y la climatología mediterránea ya ha sido destacada por Quereda et al. (2002), Miró et al. (2006) o por Sigró et al. (2014) entre otros. 


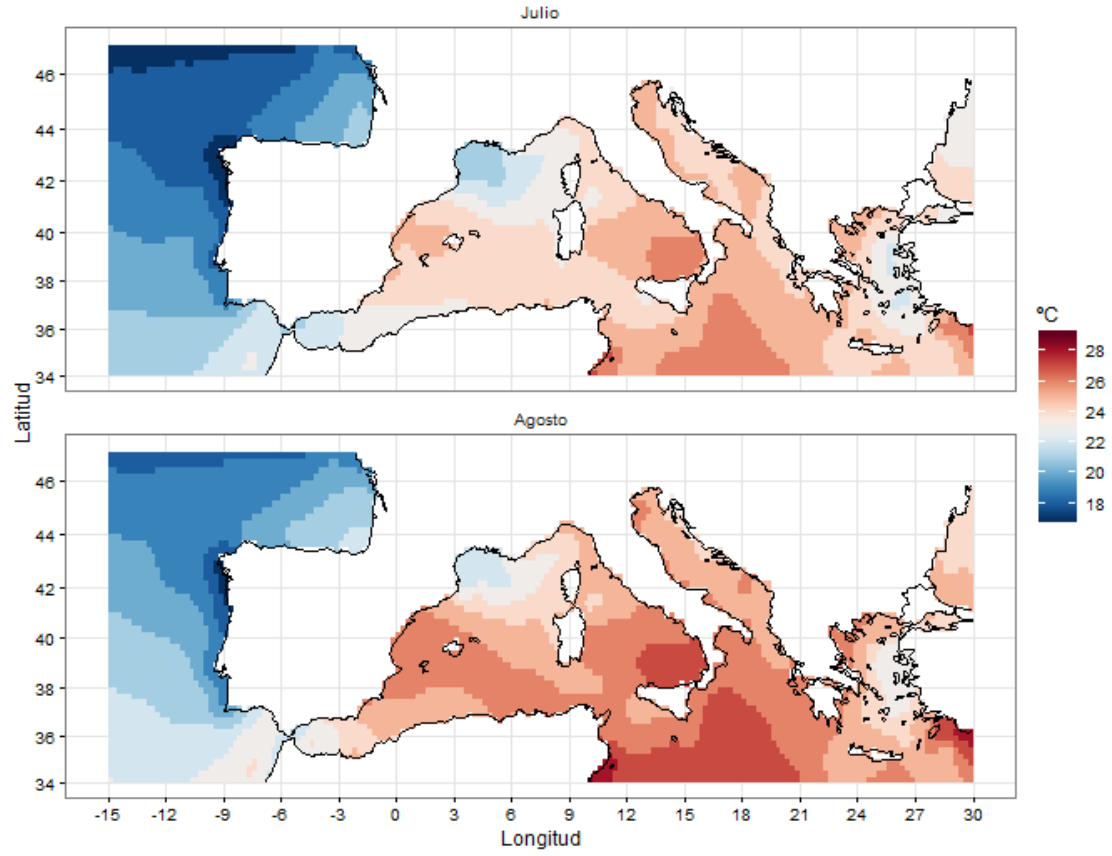

Fig. 2: Temperaturas medias de la superficie del mar Mediterráneo en julio y agosto. Fuente de datos: NOAA High Resolution SST data (NOAA/OAR/ESRL PSD), Reynolds et al., (2007).

Tras calcular el número de NC según el método descrito anteriormente, se observa cómo a diferencia de las NT todas las estaciones costeras peninsulares registran un número superior a 80 noches en las que durante más del $40 \%$ de las horas han estado por encima de los umbrales térmicos correspondientes al percentil $95 \%$ de las temperaturas mínimas (Tabla 1). El número de NC es ahora similar en la mayoría de las estaciones analizadas, debido al aumento de los valores umbrales desde Barcelona hasta Almería. En el caso de Girona, la enorme diferencia entre las 6 NT y las 60 NC se debe, por un lado, a que el percentil 95\% utilizado como umbral es algo menor que $20^{\circ}$, umbral utilizado para las NT; pero sobre todo este fuerte incremento se debe al carácter interior y más continental de la ciudad, donde las temperaturas mínimas descienden más rápidamente durante la noche, de tal forma que, aunque en las primeras horas nocturnas las temperaturas puedan ser elevadas y generar estrés térmico, en la madrugada, cuando se alcanza habitualmente la temperatura mínima, ésta ya ha descendido por debajo del umbral de $\operatorname{los} 20^{\circ}$ que designaría la noche como tropical. Un comportamiento similar fue analizado en Ourense, la ciudad más calurosa de Galicia, con un escaso número de NT pero con un elevado número de NC (Royé y Martí, 2015).

La mayor parte de esas NT y NC se concentran en los meses de julio y agosto, con valores medios comprendidos entre 25 y $30 \mathrm{NC}$ o entre 15 y $25 \mathrm{NT}$ en cada uno de los meses, especialmente en las estaciones de la costa oriental peninsular (Fig.3). Pero también durante los meses de junio y septiembre este tipo de noches calurosas son relativamente frecuentes, sobre todo en este último mes, en el que se registran una 
media de entre 15 y $20 \mathrm{NC}$ y entre 10 y $15 \mathrm{NT}$ en todos las ciudades salvo en Palma y en Girona. Si bien muy puntualmente se han registrado NC en los primeros cuatro meses del año y en los dos últimos, no es hasta mayo cuando las temperaturas nocturnas alcanzan unos valores lo suficientemente elevados como para dar lugar a noches cálidas y a situaciones de estrés térmico, que se muestran más frecuentes a partir de junio y hasta mediados de octubre. Como se puede observar en la Fig.4 es en Girona donde el periodo de riesgo de NC y NT es más corto. Al contrario que en Almería, donde se observa cómo existe probabilidad de noches cálidas durante prácticamente todo el año, si bien son muy puntuales los primeros y últimos meses del año.

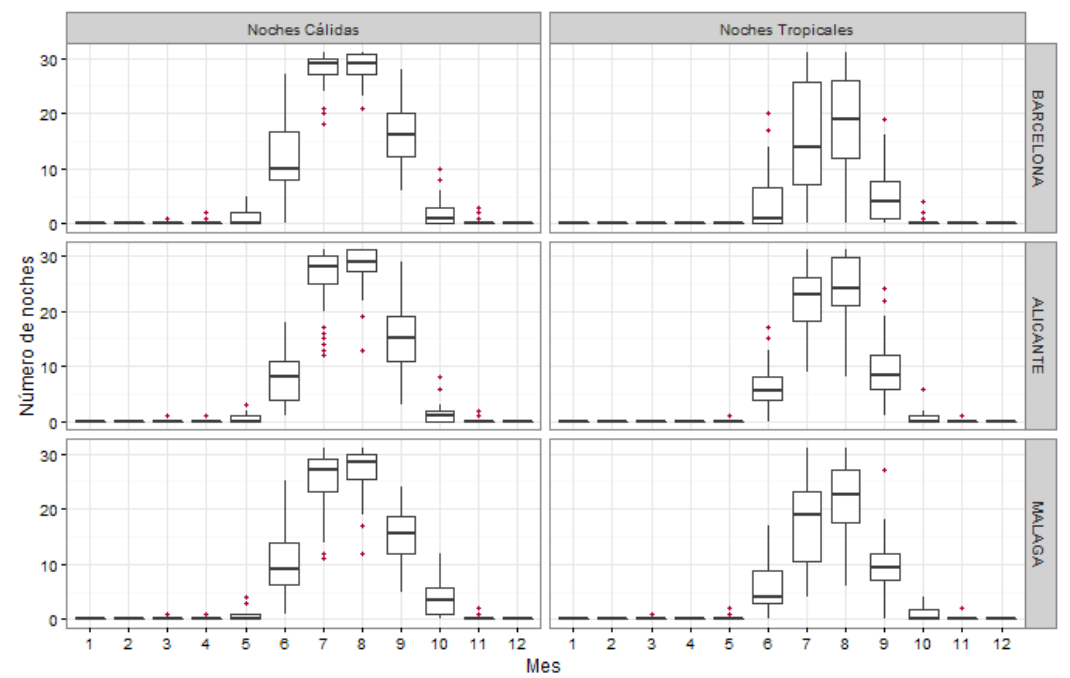

Fig. 3: Frecuencia mensual de las noches cálidas y de las noches tropicales.

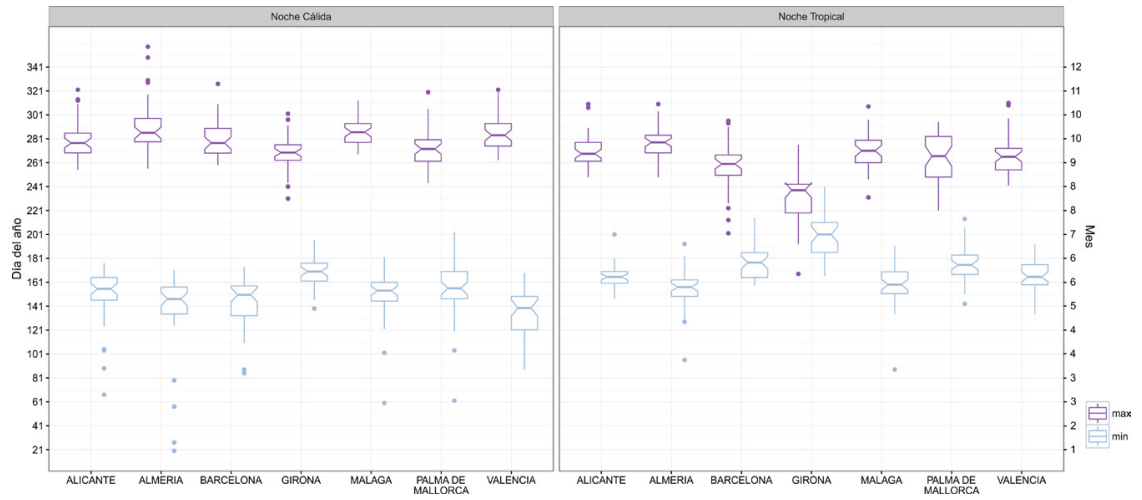

Fig. 4: Fechas de inicio y final durante el año de las noches cálidas y tropicales.

Tanto las NT como las NC han experimentado una tendencia de aumento durante el periodo de 41 años analizado (Fig. 4 y 6). En el caso de las NT los incrementos han 
sido muy fuertes en Barcelona (Tabla 2), donde han sufrido un aumento medio de 1,7 noches/año; otras ciudades como Málaga o Palma han visto también incrementado el número de NT de forma considerable (1,17 y 1 noche/año respectivamente). El incremento se sitúa en torno a 0,7 noches/año en el resto de estaciones, salvo en Girona, donde apenas varía. Las NC han experimentado igualmente fuertes tendencias de aumento, con valores que van desde los 0,5 días/año en Barcelona, pasando por los 0,96 de Valencia, hasta los 1,45 de Girona.

En un trabajo reciente sobre extremos térmicos en el litoral mediterráneo (Sigró et al., 2012) se detectaron también fuertes incrementos en las tendencias de las noches y días cálidos a partir de los inicios de los años 70. Los autores obtuvieron aumentos de las noches cálidas (percentil 90) para el periodo 1973-2005 de 10,5 noches/década en verano, de 5,6 noches en primavera y de 4,3 noches en otoño, cuando en el periodo más amplio de 1901-2005 la tendencia de aumento había sido sólo de 0,76 noches/ década.

\begin{tabular}{cccccccc}
\hline & Alicante & Almería & Barcelona & Girona & Málaga & Palma & Valencia \\
\hline NC & 0,62 & $0,23^{*}$ & 0,51 & 1,45 & 1,0 & 0,68 & 0,96 \\
\hline NT & 0,65 & 0,72 & 1,76 & 0,09 & 1,17 & 1,0 & 0,68 \\
\hline
\end{tabular}

Tab. 2: Tendencias anuales de las noches cálidas y tropicales (Sen's Slope) para el periodo 1974-2015, con $p \leq 0,01 ;{ }^{*} p=0,11$.

Para explicar dichas tendencias tenemos que hablar de una multicausalidad. Por un lado el incremento de las temperaturas del aire asociadas al calentamiento global que afecta a toda la península, y especialmente a las regiones mediterráneas (Quereda et al., 2000), con mayor intensidad desde los años 70, cuando se constata el incremento más riguroso observado en las temperaturas españolas (Brunet et al., 2007). La transformación del territorio, los cambios de usos del suelo, el fuerte desarrollo urbanístico que ha tenido lugar en las últimas décadas en España en general, y en muchas áreas del litoral mediterráneo en particular, constituyen otro posible factor explicativo de los incrementos de las noches cálidas, si tenemos en cuenta los efectos directos que dichos cambios tienen sobre las temperaturas del entorno. Quereda et al. (2000) ya hablaban de una tendencia de calentamiento significativa en muchas ciudades de la España mediterránea, y ponían especial acento en el efecto urbano y en el cambio de usos del suelo en el entorno de las estaciones meteorológicas como factores a tener en cuenta a la hora de explicar parte de dichas tendencias.

Otro importante factor es el incremento constatado de la temperatura superficial del mar Mediterráneo. Ya vimos anteriormente cómo el gradiente de la SST del Mediterráneo occidental influía, junto a la latitud, en la variación espacial de las noches tropicales. Sigró et al. (2012) señalan a la SST del Mediterráneo como principal factor de rozamiento asociado a los cambios en los extremos de temperatura, y es en verano cuando obtienen los mayores coeficientes de correlación significativos entre ambas variables. Miró et al. (2006) señalan también un incremento en la persistencia de días cálidos en el verano en la región valenciana, relacionándolos con la temperatura superficial del mar Mediterráneo. En un reciente trabajo, Shaltout y Omstedt (2014) obtienen una tendencia de calentamiento de la SST en el Mediterráneo occidental de 
$0,33^{\circ}$ por década entre 1982 y 2012 , y señalan la posibilidad de escenarios con incrementos de $2^{\circ} \mathrm{C}$ a finales del siglo XXI. Igualmente, en sendos estudios de Vargas et al. (2010) y de la Agencia Europea de Medioambiente (2015) se constata un incremento medio de casi $1^{\circ} \mathrm{C}$ para el mar Mediterráneo entre 1975 y 2010, que puede explicar, junto a los factores anteriores, las fuertes tendencias de aumento que han sufrido las noches calurosas en todo el Mediterráneo occidental.

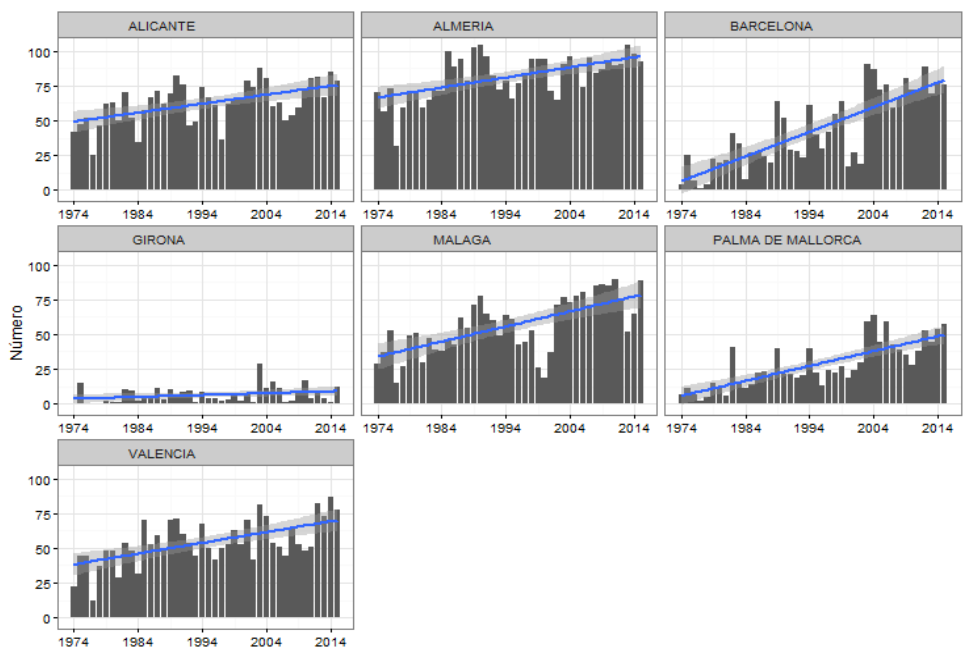

Fig. 5: Evolución temporal de las noches tropicales entre 1974 y 2015.

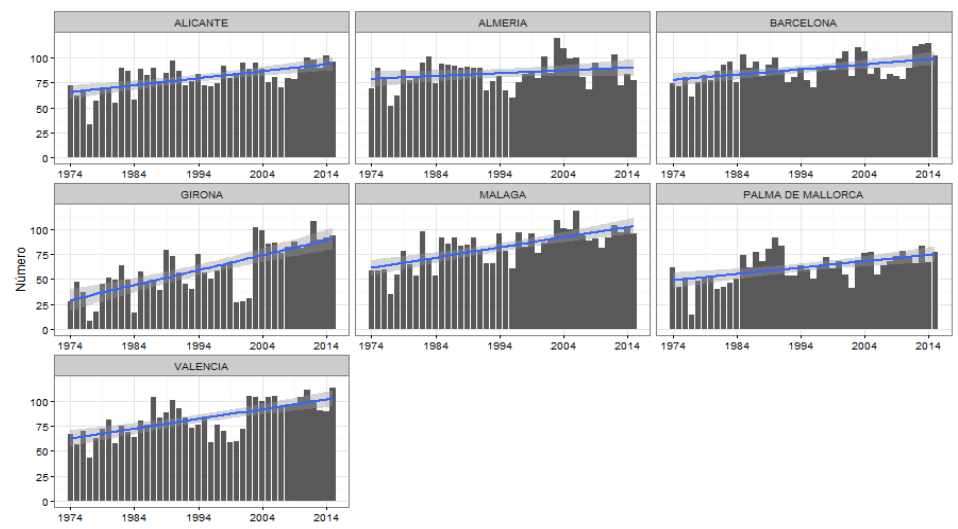

Fig. 6: Evolución temporal de las noches cálidas entre 1974 y 2015.

\section{CONCLUSIONES}

Con la aplicación del método propuesto para la cuantificación de noches cálidas se ha comprobado que un importante número de noches con posible estrés térmico en 
la primera mitad de la noche queda oculto si solamente se tienen en cuenta las temperaturas mínimas como indicador. La utilización de datos horarios permite valorar con más detalle las características térmicas de las noches, especialmente en los meses estivales, pudiendo así evaluar con más precisión el riesgo para el bienestar y la salud de la población. Hay que tener en cuenta, además, que son las primeras horas de la noche y la primera fase del sueño la que se describe como la más sensible y la que acumula las mayores alteraciones por estrés térmico. Con la utilización de un umbral relativo de la temperatura mínima, el percentil 95, se han tenido en cuenta las características termométricas del clima local y la aclimatación de la población.

Los resultados del análisis han constatado, en primer lugar, una elevada frecuencia de NT en el área de estudio, que aumenta de norte a sur en toda la franja litoral oriental; en segundo lugar una gran diferencia entre el número de NC y el de NT, de más del doble en el caso de Girona y Barcelona; en tercer lugar una concentración de estas noches calurosas entre los meses de junio y septiembre, especialmente en julio y agosto, cuando en la mayor parte del litoral mediterráneo español se registran como media entre 25 y $30 \mathrm{NC}$ y entre 15 y $25 \mathrm{NT}$. El análisis de los datos en el periodo 1974-2015 muestra también un aumento continuado de la frecuencia de NT y de NC en todas las estaciones analizadas, especialmente destacado en Barcelona, en Málaga o en Palma. El incremento general de las temperaturas, más intenso desde la década de los 70, los cambios de uso del suelo en el entorno de los observatorios meteorológicos y el incremento de la SST del Mediterráneo explican esas tendencias de aumento.

La menor latitud y la proximidad al litoral están directamente relacionados con la mayor persistencia del calor y del estrés térmico durante las noches cálidas. En zonas más continentales, como el caso de Girona, la persistencia del calor es, sin embargo, menor, debido al más rápido enfriamiento de las temperaturas nocturnas.

\section{REFERENCIAS}

Alexander, L. V., Zhang X., et al., (2006). Global observed changes in daily climate extremes of temperature and precipitation. Journal of Geophysical Research, 111.

Andrade, H. y Alcoforado, M.-J. (2008). Microclimatic variation of thermal comfort in a district of Lisbon (Telheiras) at night. Theor. Appl. Climatol., 92, 225-237.

Buguet, A. (2007). Sleep under extreme environments: Effects of heat and cold exposure, altitude, hyperbaric pressure and microgravity in space. Journal of the Neurological Sciences, 262, 145-152.

Bobb, J.F.; Peng, R.D.; Bell, M.L. y Dominici, F. (2014). Heat-Related Mortality and Adaptation to Heat in the United States. Environmental Health Perspectives, 122:811-816.

Brunet, M.; Jones, M.; Sigró, P.; Saladié, J. y Aguilar, O. (2007). Temporal and spatial temperature variability and change over Spain during 1850-2005. Journal of Geophysical Research-Atmospheres, 112.

Coumou, D. y Rahmstorf, S. (2012). A decade of weather extremes. Nature Climate Change, 2, 491-496.

Coumou, D. y Robinson, A. (2013). Historic and future increase in the global land area affected by monthly heat extremes. Environ. Res. Lett., 8. 
Donat, M.G., et al., (2013). Updated analyses of temperature and precipitation extreme indices since the beginning of the twentieth century. Journal of Geophysical Research: Atmospheres, 118, 1-16.

DWD (2013): Deutscher Wetterdienst - Wetterlexikon (Servicio Alemán de Meteorología - enciclopedia meteorológica), http://www.dwd.de/bvbw/ appmanager/bvbw/dwdwwwDesktop?_nfpb=true\&_pageLabel=dwdwww menu2_wetterlexikon\&_nfls=false, 25/09/2013.

EEA Report (2012). Urban adaptation to climate change in Europe: Challenges and opportunities for cities together with supportive national and European policies. Nr 2. Copenhagen: European Environment Agency.

Fischer, E.M. y Schär, C. (2010). Consistent geographical patterns of changes in highimpact European heatwaves, Nature Geoscience, 3, 398-403.

Gronlund, C.J.; Zanobetti, A.; Schwartz, J.D.; Wellenius, G.A. and O’Neill, M.S. (2014). Heat, Heat Waves, and Hospital Admissions among the Elderly in the United States, 1992-2006. Environmental Health Perspectives, 122, 1187-1192.

Hajat, S. y Kosatky, T. (2010). Heat-related mortality: a review and exploration of heterogeneity. J Epidemiol Community Health, 64, 753-760.

Haskella E.H.; Palcaa J.W.; Walkera J.M.; Bergera R.J.; Hellera H.C. (1981). The effects of high and low ambient temperatures on human sleep stages. Electroencephalography and Clinical Neurophysiology, 51, 494-501.

Hong Kong Observatory (2012): http://www.hko.gov.hk/cis/regione.htm, 25/09/2013.

IPCC (2014). Impacts, Adaptation and Vulnerability. Working Group II Contribution to AR5.

Koppe, C.; Kovats, S.; Jendritzky, G. y Menne, B. (2004). Heat-waves: risks and responses, WHO Regional Office for Europe.

Laaidi, K.; Zeghnoun, A.; Dousset, B.; Bretin, P.; Vandentorren, S.; Giraudet, E. y Beaudeau, P. (2012). The Impact of Heat Islands on Mortality in Paris during the August 2003 Heat Wave. Environmental Health Perspectives, 120, 254-259.

Lisa, V.; et al., (2009). Climate extremes: progress and future directions. International Journal of Climatology, 29, 317-319.

Miró, J.; Estrella, M.J. y Millán, M. (2006): Summer temperature trends in a Mediterranean area (Valencia región). International Journal of Climatology, 26, 1051-1073.

Nastos P. T. y Matzarakis A. (2008). Human-Biometeorological effects on sleep disturbances in Athens, Greece: A Preliminary Evaluation. Indoor Built Environment, 17, 535-542.

Okamoto-Mizuno K. y Mizuno K. (2012). "Effects of thermal environment on sleep and circadian rhythm", Journal of Physiological Anthropology, 31:1:14.

Okamoto-Mizuno, K.; Tsuzuki, K. y Mizuno K. (2005). Effects of humid heat exposure in later sleep segments on sleep stages and body temperature in humans. Int J Biometeorol, 49, 232-237. 
Quereda, J.; Gil Olcina, A.; Pérez, A.; Olcina, J.; Rico, A. y Montón, E. (2000). Climatic warming in the spanish Mediterranean: natural trend or urban effect. Climatic Change, 46, 473-483.

Quereda, J.; Montón, E. y Escrig, J. (2002). Las interacciones atmósfera-mar en la climatología mediterránea del 2001. Investigaciones Geográficas, 29, 23-38.

Reynolds, R.W.; Smith, T.M.; Liu, C.; Chelton, D.B.; Casey, K.S.; Schlax, M.G. (2007). Daily High-Resolution-Blended Analyses for Sea Surface Temperature. J. Climate, 20, 5473-5496.

Royé, D. y Martí, A. (2015). Análisis de las noches tropicales en la fachada atlántica de la Península Ibérica. Una propuesta metodológica. Boletín de la Asociación de Geógrafos Españoles, 69, 351-368.

Royé, D. (2015). Another Approach for Tropical Nights: Case Studies of Faro, Lisbon and Porto. En: Pina, H. \& Martins F., The overarching issues of the european space: spatial planning and multiple paths to sustainable and inclusive development, Faculdade de Letras da Universidade do Porto, 269-283.

Russo, S. y Sterl, A. (2011). Global changes in indices describing moderate temperature extremes from the daily output of a climate model. Journal of Geophysical Research: Atmospheres, 116.

Shaltout, M. y Omstedt, A. (2014). Recent sea surface temperature trends and future scenarios for the Mediterranean sea. Oceanología, 56, 411-443.

Sigró, J.; Brunet, M. y Aguilar, E. (2014). Los extremos térmicos en el litoral mediterráneo: evolución y factores de forzamiento. Territoris, 8, 265-281.

Vargas, M.; García, M. y Moya, F. (2010). Cambio climático en el Mediterráneo español. Instituto Español de Oceanografía. $\mathrm{M}^{\mathrm{o}}$ Ciencia e Innovación. Madrid.

Vincent, L.A.; Peterson, T.C.; et al., (2005). Observed Trends in Indices of Daily Temperature Extremes in South America 1960-2000. AMS Journal of Climate, 18, 5011-5023.

WMO (2009). Analysis of extremes in a changing climate in support of informed decisions for adaptation. World Meteorological Organization. Geneva. WCDMPNo. 72.

Ye, X.; Wolff, R.; Yu, W.; Vaneckova, P.; Pan, X. and Tong, S. (2012). Ambient Temperature and Morbidity: A Review of Epidemiological Evidence. Environ Health Perspect, 120, 19-28. 